\title{
Recommendations for cardiovascular evaluation in athletes - a viewpoint
}

\section{CARDIOLOGY / PREPARTICIPATION / SPORTS MEDICINE / TEAM MANAGEMENT}

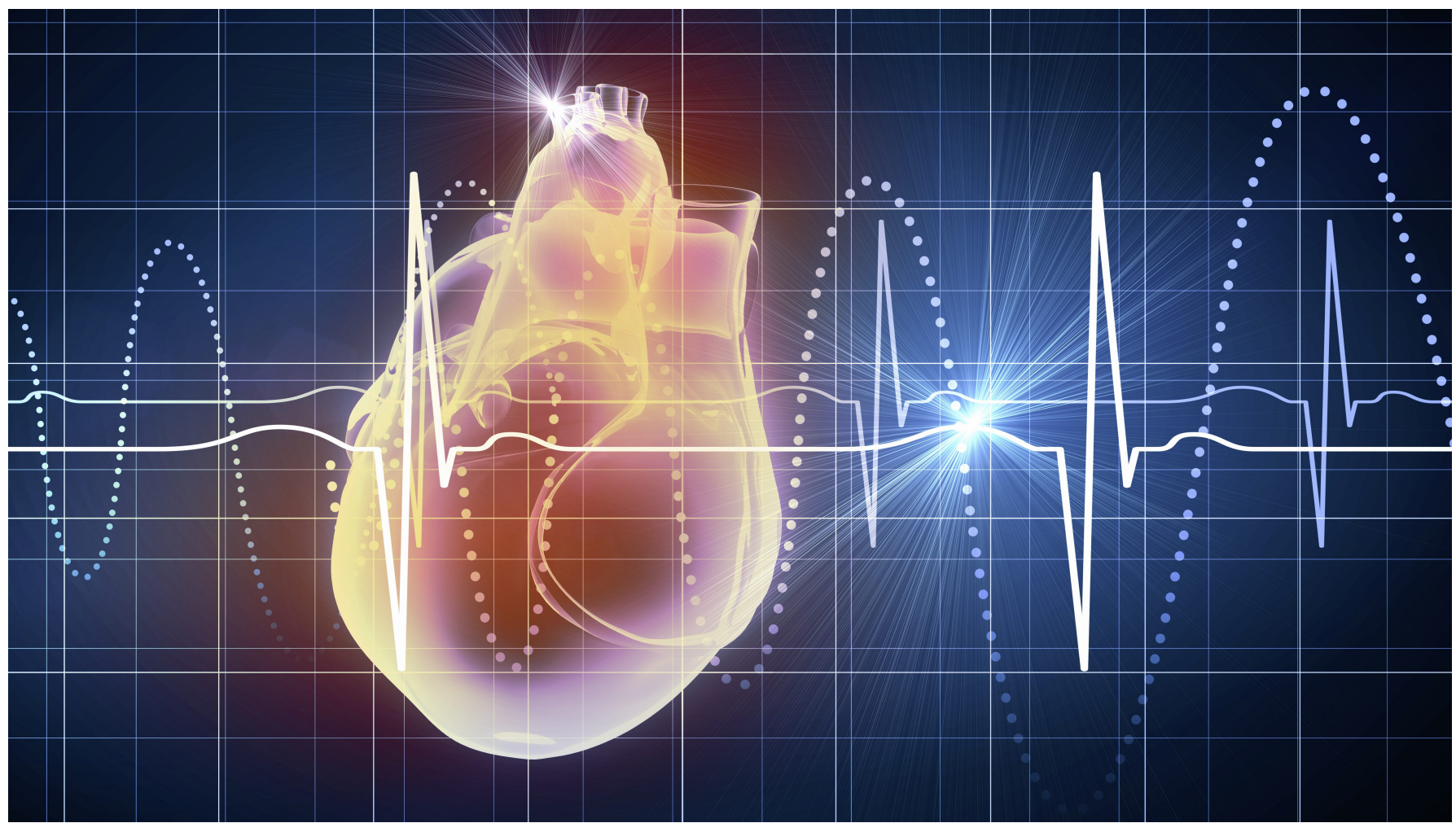

Kissel CK, Niederseer D, Bohm P, Schmied CM

University Heart Centre, Dept. of Cardiology, University Hospital Zurich, Switzerland

\begin{abstract}
Traditionally, cardiovascular screening has been recommended mostly for competitive athletes younger than 35 years. The perception that only young competitive athletes at top level are at increased risk, has changed in the last years. Theoretically, we advocate a voluntary cardiovascular screening for all athletes who exercise vigorously, independent of their competitive status, and age. Although, this should be based on an individual estimation of the athlete's risk. Physical examination, medical history and an ECG should be the baseline investigations for all athletes. Ideally, an echocardiography should be performed once at the beginning of the athletic career to rule out congenital coronary and valvular abnormalities-although addition of an echocardiography has not yet been established routinely due to lacking evidence. We advocate to repeat the ECG every 1-2 years in athletes $<30-35$ years. For older athletes, we recommend to
\end{abstract}


assess their individual CV risk profile (particularly with inclusion of lipid profile) and their previous training history. Depending on the assessment, further investigations should be implemented such as an exercise stress test and/or Coronary CT scan.

\section{Zusammenfassung}

Bisher wurde üblicherweise ein kardiovaskuläres Screening v.a. für junge kompetitive Athleten unter 35 Jahren empfohlen. Die Vorstellung, dass nur junge Athleten, welche an Wettkämpfen teilnehmen, ein erhöhtes Risiko haben könnten, hat sich in den letzten Jahren jedoch gewandelt. Theoretisch wäre ein freiwilliges, kardiovaskuläres Screening für alle Athleten, welche sich intensiv sportlich betätigen, unabhängig von Alter und ob sie an Wettkämpfen teilnehmen, wünschenswert - in Abhängigkeit der individuellen Risikoabschätzung für den Athleten. Körperliche Untersuchung, Anamnese und EKG sollten die Basisuntersuchung für alle Athleten darstellen. Eine Echokardiographie wäre idealerweise am Beginn der sportlichen Karriere wünschenswert, um kongenitale und valvuläre Erkrankungen auszuschliessen auch wenn die Echokardiographie bisher, aufgrund fehlender Daten, noch nicht routinemässig für Screeninguntersuchungen etabliert wurde.

Wir befürworten des Weiteren, dass EKGs bei Athleten unter 30-35 Jahren alle 1-2 Jahre wiederholt werden sollten. Für ältere Athleten empfehlen wir, das individuelle kardiovaskuläre Risiko abzuschätzen unter Einbezug von aktuellen Cholesterinwerten und Trainingsanamnese. In Abhängigkeit des individuellen Risikos empfehlen wir weitere Untersuchungen wie Belastungs-EKG und/oder Coronar-CT.

\section{Introduction}

Sport and exercise activities are recommended by expert panels to promote and maintain cardiovascular (CV) health and to prevent disease [1]. However, if an underlying cardiac condition exists, sport can potentially trigger a life-threatening ventricular arrhythmia and cause sudden cardiac death (SCD). Not the sporting activity per se increases the risk of SCD but the combination of underlying CV disease with sport. Therefore, most international professional societies recommend a cardiovascular evaluation of athletesalthough protocols vary between the different countries [2-4].

The incidence of SCD is difficult to determine because most registries are retrospective. Also, determination of the exact number of deaths and the number of athletes who actually participated (as denominator) can be challenging [2]. Overall, the incidence of SCD appears to be low and in the range of 0,7 to 2,3 out of 100000 athletes [5-8]. A retrospective study also confirmed a low incidence of sportsrelated SCD in Switzerland [9]. However, data from Italy clearly showed a reduction of the incidence of SCD by pre-participation screening [10].

The goal of this "viewpoint" article is to review the current data and to provide aspects and perspectives for a possible "Swiss approach" of a screening concept.

\section{Who to screen?}

Previous recommendations have focused on so-called competitive athletes. However, the definition of the term "competitive" is debatable and it is increasingly recognized that recreational athletes also push to their limits to improve their performance and that they also often "engage in regular, systematic and often 
intense training with high CV demands", which was originally the definition for competitive athletes [11]. Furthermore, a recent study from Denmark has shown that the risk of SCD is similar in competitive and non-competitive athletes [12]. Other studies have shown that not only the competitive status, but also factors such as baseline fitness, ethnicity, male sex, the nature and intensity of athletic activity may play a role in increasing the risk for sports-related SCD [3,11,12]. Therefore, we advocate that theoretically all athletes who engage in regular, vigorous exercise, independent of the competitive status, should undergo CV evaluation. Nevertheless, evidence for non-selective "mass screening" is limited and how to estimate the athlete's risk is still a matter of debate. We think that screening should be voluntary. However, this mandates adequate education and information of the community and availability of screening programs and dedicated and skilled physicians in this field.

The extent of the baseline CV evaluation should depend on the age of the athletes since the etiology of SCD changes with increasing age.

In very young athletes ( $<12$ years), $\mathrm{CV}$ evaluation should be guided by medical and family history, as well as by physical examination and training intensity. Incorporating an ECG into the routine pediatric evaluation was shown to be feasible [13], and might be worth consideration. However, most children only start to participate in more strenuous exercise above the age of 12 years. In young athletes (age < 30 to 35 years), SCD is mostly associated with an inherited CV conditions, such as inherited primary arrhythmia syndromes (e.g. Brugada, long QT syndrome, catecholaminergic polymorphic ventricular tachycardia) [14], or inherited cardiomyopathies (e.g. hypertrophic cardiomyopathy (HCM), arrhythmogenic right ventricular cardiomyopathy (ARVC), dilated cardiomyopathy (DCM)). Coronary artery anomalies pose another congenital cause of SCD, as well as ventricular pre-excitation. With increasing age (>25 to 35 years), acquired CV conditions become more predominant causes of sports-related SCD in the general population. Foremost, coronary artery disease (CAD) is the most common etiology, but myocarditis has also been shown to play a role $[9,12,15,16]$. In view of the different etiologies, different screening protocol should be applied in our opinion.

\section{How to?}

\section{All athletes-ECG}

The basis of every CV evaluation should be a standardized medical questionnaire, which covers typical symptoms suggestive of a CV condition (i.e. chest pain, dyspnea, palpitations, dizziness, syncope) but also family history in regard to CV disease and especially SCD. Furthermore, a focused physical examination should be conducted (inspection for Marfanoid features [17], blood pressure on both arms, cardiac auscultation for murmurs). The sensitivity to detect a CV abnormality is in between $20-45,5 \%$ with this approach [18,19].

Sensitivity of detection a relevant CV condition improves substantially to $>90 \%$ by incorporating an ECG into the evaluation protocol [19]. A majority of inherited arrhythmia syndromes, which carry an increased risk for SCD, can be detected by resting ECG [2,20,21]. In addition, pre-excitation syndromes can be detected in a resting ECG in the majority of cases. Most cardiomyopathies also present with an abnormal resting ECG, e.g. an abnormal ECG pattern can be detected in up to $90 \%$ of HCM $[22,23]$ and $>80 \%$ of ARVC patients [24]. Unspecific changes can also be found in patients with DCM, myocarditis and LVNC [2,25]. 
Together these conditions account for two thirds of SCD in young athletes [14]. Sensitivity of the ECG is not perfect but adding an ECG increases the likelihood of detection significantly, and has been shown to decrease SCD among young athletes after nation-wide implementation in Italy [10,19]. One major concern is, or was, specificity. A false positive rate of $5-10 \%$ has been reported [26,27]. However, with the newest "International Criteria” for ECG interpretation, sensitivity and specificity has been further increased [28]. This improvement further reduced the false-positive rate, and the cost of follow-up investigations [29]. Thus, from an evidence based point of view, it is no more debatable whether an ECG should be included in the screening of young athletes [29].

In regard to screening frequency, we recommend to repeat ECGs every 1-2 years in these young athletes because some cardiomyopathies only become apparent with increasing age and training loads [2,24,30,31], and some primary arrhythmia syndromes vary in their appearance on the resting ECG [2].

\section{The role of the echocardiography}

Another drawback of the ECG is that it fails to detect coronary artery anomalies, as well as aortopathies. The detection of relevant valvulopathies in clinical examination is challenging (e.g. bicuspid aortic valve, mitral valve prolapse), although these diseases are relevant particularly in an athletic population. Congenital coronary anomalies have an incidence of $0,5 \%$ to $2 \%$, and are the second most common cause of SCD in young athletes in some series [32,33]. Echocardiography can detect the origin of the coronary arteries in the majority of cases (left main stem 97\%, 80\% RCA) [34]. Furthermore, congenital abnomalities such as bicuspid aortic valve, aortopathies and mitral valve prolapse can be detected, which can pose an increased risk depending on the sporting discipline. Due to its advantages, some major sporting bodies have implemented echocardiography in their mandated CV evaluation protocols. The Fédération Internationale de Football Association (FIFA) and the Union of European Football association (UEFA), as well as the Union Cycliste Internationale (UCI) mandate an echocardiography in the screening of their athletes [2]. Concerns against performing an echocardiography are mostly additional cost, and an increased rate of false-positive findings due to training-induced changes.

We advocate an one-time ecocardiographic evaluation at the beginning of the athletic career to rule out a congenital coronary or valvular abnomality. Moving forward in their career to "master athlete status", we suggest another echocardiography, mainly to detect dilation of the aorta and to assess cardiovascular changes that develop through life-long atletic activity. As such, atrial fibrillation due to atrial dilation and right heart fatigue, a condition of the right ventricle, are relevant diseases in that particular group of athletes. Preferably, echocardiography should be performed onsite of the CV evaluation to reduce associated cost and time delays until the athlete can be cleared [35].

\section{Older athletes ( $>\square 25$ to 35 years) and their risk for coronary plaque rupture}

With increasing age, coronary artery disease (CAD) becomes by far the most common cardiac cause of sports-related sudden death in athletes $[9,15,16]$. Prevalence of artherosclerosis increases with age and presence of conventional CV risk factors. Assessment of CV risk factors therefore appears self-evident. In this regard, the SCORE system of the European Society of Cardiology (ESC) helps to estimate the 10-yearrisk of fatal CV disease (https://www.escardio.org/Education/Practice-Tools/CVD-prevention-toolbox/SCORERisk-Charts) [36]. These recommendations have massively improved in their last version, as they allow to 
further specify risk calculation (e.g. by estimating "lifetime risk" and by integrating further risk factors as ethnic background) [37]. An individualized risk estimation is particularly crucial in an athletic population where cardiovascular risk is often underestimated.

For senior individuals, the ESC guidelines recommend further CV evaluation depending on age, CV risk profile by SCORE, prior physical activity and planned training intensity [38]. Whereas the AHA guidelines recommend CV evaluation of all men $>40$ years and all women $>50$ years with one additional risk factor who take part in competitive sports [38]. Both societies recommend an exercise test (ET) as primary additional test on top of ECG, physical examination and medical history. Appart from CAD diagnosis, exercise ECG testing bears the advantage to assess blood pressure response, physical performance and arrhythmias during exercise. However, the sensitvity of exercise ECG tests to detect ischemia is limited (68\%), and the specificity is only $80 \%$ [40]. Furthermore, ET will not detect non-obstructive CAD which is not flow-limiting. Psychological and physical stress potentially carries an increased risk for plaque rupture, likely mediated by increased sympathetic and hormonal activation [2,38,41]. To determine the individual risk, we recommend that a lipid profile should be determined in all patients above 25 years. Cholesterol levels are also a part of the SCORE risk calculator. However, one of the disadvantages of the SCORE risk calculator is that it does not take into account a positive family history, which has a crucial impact on the life-time risk. The SCORE risk calculator only estimates the predicted 10-years risk of a CV event, which is a good tool to identify people who benefit from preventive drug therapy. However, some people may be at low risk ( $<5 \%)$ to develop a CV event in the next 10 years but carry an increased life-time risk [37,43]. This is especially true for women and athletes, who have a positive family history or dyslipidemia. In these populations, estimation of life-time risk can help to communicate the high-risk situation and may advocate a computed tomography coronary angiography (CTCA) for further risk stratification in mature athletes. CTCA has the best ability to risk-stratify and re-classify risk categories, whereas other tools such as carotid ultrasound do not [43].

Furthermore, it has recently been shown that male athletes who have been exercising on high levels for an extended period of time ( $>10$ years, $\geq 4 \mathrm{hrs} / \mathrm{wk}$ ), have a higher prevalence of atherosclerotic plaques than sedentary individuals [44]. This non-obstructive CAD is not accurately detected by exercise ECG, and CTCA reveals a relevant burden in one quarter of veteran athletes aged $>40$ years [45]. Therefore, one might consider a CTCA in veteran athletes, particularly if their CV risk profile is high. Nevertheless, it is important to discuss possible consequences of an increased plaque burden with the athlete before undergoing CTCA imaging. Detection of coronary sclerosis might prompt a recommendation for secondarypreventive statin therapy and life-style changes, and might have a relevant psychological impact on the athlete [46].

The ESC consensus statement also recommends to take into consideration the previous exercise history, as well as the planned intensity of training [38]. We advocate that mature athletes who have been sedentary for a while should undergo CV evaluation, since a lower fitness level correlates with an increased risk for CV events [38,47]. However, in our opinion the decision whether to undergo CV evaluation in sedentary subjects should not only be based on the planned intensity of training. The perception of training intensity can be very subjective, and even low intensity activities can include short bursts of higher intensity, or can be negatively influenced by unfavorable environmental conditions (e.g. heat). Older athletes should therefore undergo a basic CV evaluation (ECG, PE, history, lipid profile), and additional tests such as ET or CTCA based on the individual risk. 
Athletes who have been exercising regularly but who plan to substantially increase their exercise intensity also should undergo a basic CV evaluation with further testing (ET or CTCA) after risk stratification and discussion with the athlete [38].

In summary, we recommend a basic CV evaluation in all sedentary individuals ( $>25$ years) who plan to start exercising, or in all active athletes who will substantially increase their training intensity and ambitions. CV evaluation should include an ECG, physical exam (with blood pressure measurement), medical history, and a lipid profile. Depending on symptoms and the individual risk (lifetime and 10-year, with inclusion of family history), the patient should be counseled to undergo further testing with exercise ECG and/or CTCA for CV risk stratification. In athletes over 30 to 35 years who exercise regulary, we recommend to repeat a basic CV evaluation every 3 to 5 years.

Figure 1 gives an overview of an possible screening strategy throughout an athlete's career. Although some parts are not "evidence based", it should serve as a basis for further discussion. And it should once more underline that screening of athletes should always be individualized.

\section{Conclusion}

In the past, CV screening has been recommended mostly for competitive athletes younger than 35 years. The perception that only young competitive athletes on a top level are at increased risk, has changed in the last years. We advocate a CV screening for all athletes who exercise vigorously, independent of their competitive status, and age. Physical examination, medical history and an ECG should be the baseline investigations for all athletes. Ideally, an echocardiography should be performed once at the beginning of the athletic career to rule out congenital coronary and valvular abnormalities. Although the addition of an echocardiography has not been routinely established yet due to lacking evidence. We advocate to repeat the ECG every 1-2 years in athletes $<30$ to 35 years. For older athletes, we recommend to assess their individual and specific CV risk profile (particularly with an additional cholesterol assessment) and their previous training history. Depending on the assessment, further investigations should be implemented such as an exercise stress test and/or CTCA.

Implementing these recommendations needs widespread expertise in interpretation of the athlete's ECG by standardized criteria [28]. This requires specialist training because even amongst cardiologist the interobserver agreement is suboptimal, which often leads to (unnecessary) further investigations [48]. However, even with an eloborate screening protocol, the risk of SCD cannot be completely eliminated and some patients at risk will not be identified, e.g. athletes with a scar/fibrosis after myocarditis. Therefore, it is crucial that emergency response plans during training and competition are well thought-out and that cardiopulmonary resuscitation is practiced on a regular basis. Furthermore, the wide-spread availability of automatic external defibrillators is equally important as the most sophisticated pre-participation screening. 


\section{0-12 years}

Risk estimation: Clinically, medical history (i.e. family history)

Screening modality: Individualised (clinical examination, imaging)

>12-14 years (beginning of regular vigorous exercise)

Risk estimation: Voluntary systematic screening ("mass screening»?)

Screening modality: Medical history, clinical examination, ECG

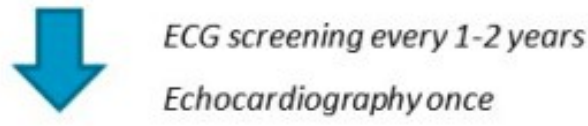

> 30 - 35 years ("older athlete»)

Risk estimation: Individualised, based on specified risk SCORE, risk modifiers and training history Screening modality: History, clinical examination, ECG, +/- exercise test / CTCA

\section{> 45-50 years ( $«$ master athlete $»)$}

Regular risk estimation every $3-5$ years

Risk estimation: Individualised, based on specified risk SCORE, risk modifiers and training intensity

Screening modality: History, clinical examination, ECG, echocardiography +/- exercise test, CTCA,

\section{Corresponding author}

Christine K. Kissel

University Heart Centre

Dept. of Cardiology

University Hospital Zurich

Rämistrasse 100, 8091 Zürich

Phone: +41-44-2553327

Email: Christine.Kissel@usz.ch

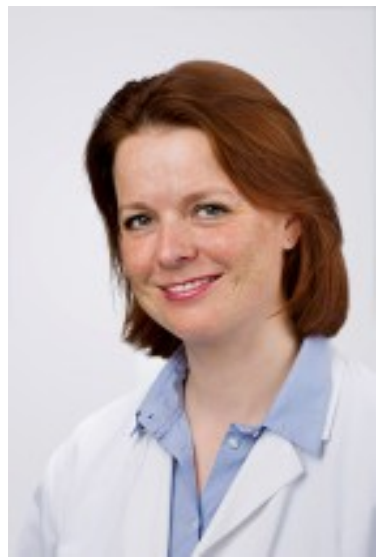




\section{References}

1. Perk J, De Backer G, Gohlke H, et al. European Guidelines on cardiovascular disease prevention in clinical practice (version 2012). The Fifth Joint Task Force of the European Society of Cardiology and Other Societies on Cardiovascular Disease Prevention in Clinical Practice (constituted by representatives of nine societies and by invited experts). Eur Heart J 2012;33:1635-1701.2012/05/05.DOI:10.1093/eurheartj/ehs092.

2. Mont L, Pelliccia A, Sharma S, et al. Pre-participation cardiovascular evaluation for athletic participants to prevent sudden death: Position paper from the EHRA and the EACPR, branches of the ESC. Endorsed by APHRS, HRS, and SOLAECE. European Journal of Preventive Cardiology 2017;24:41-69. DOI:0.1177/2047487316676042.

3. Corrado D, van-Buuren F, Mellwig KP, et al. Cardiovascular pre-participation screening of young competitive athletes for prevention of sudden death: proposal for a common European protocol: Consensus Statement of the Study Group of Sport Cardiology of the Working Group of Cardiac Rehabilitation and Exercise Physiology and the Working Group of Myocardial and Pericardial Diseases of the European Society of Cardiology. European Heart Journal 2005;26:516-524. DOI:10.1093/eurheartj/ehi108.

4. Maron BJ, Levine BD, Washington RL, et al. Eligibility and Disqualification Recommendations for Competitive Athletes With Cardiovascular Abnormalities: Task Force 2: Preparticipation Screening for Cardiovascular Disease in Competitive Athletes: A Scientific Statement From the American Heart Association and American College of Cardiology. Circulation 2015;132:e267-272. 2015/11/04.DOI:10.1161/CIR.0000000000000238.

5. Corrado D, Basso C, Rizzoli G, et al. Does sports activity enhance the risk of sudden death in adolescents and young adults? J Am Coll Cardiol 2003;42:1959-1963.2003/12/10.

6. Maron BJ, Doerer JJ, Haas TS, et al. Sudden Deaths in Young Competitive Athletes. Circulation 2009;119:1085-1092.DOI:doi:10.1161/CIRCULATIONAHA.108.804617.

7. Landry $\mathrm{CH}$, Allan KS, Connelly KA, et al. Sudden Cardiac Arrest during Participation in Competitive Sports. N Engl J Med 2017;377: 1943-1953.2017/11/16.DOI:10.1056/NEJMoa1615710.

8. Mohananey D, Masri A, Desai RM, et al. Global Incidence of Sports-Related Sudden Cardiac Death. J Am Coll Cardiol 2017;69: 2672-2673.2017/05/27.DOI:10.1016/j.jacc.2017.03.564.

9. Asatryan B, Vital C, Kellerhals C, et al. Sports-related sudden cardiac deaths in the young population of Switzerland. PloS one 2017;12: e0174434-e0174434.DOI:10.1371/journal.pone.0174434.

10. Corrado D, Basso C, Pavei A, et al. Trends in sudden cardiovascular death in young competitive athletes after implementation of a preparticipation screening program. JAMA 2006;96:1593-1601.2006/10/05. DOI:10.1001/jama.296.13.1593.

11. Maron BJ, Thompson PD, Ackerman MJ, et al. Recommendations and considerations related to preparticipation screening for cardiovascular abnormalities in competitive athletes: 2007 update: a scientific statement from the American Heart Association Council on Nutrition, Physical Activity, and Metabolism: endorsed by the American College of Cardiology Foundation. Circulation 2007;115:1643-1455. 2007/03/14.DOI:10.1161/CIRCULATIONAHA.107.181423.

12. Risgaard B, Winkel BG, Jabbari R, et al. Sports-related sudden cardiac death in a competitive and a noncompetitive athlete population aged 12 to 49 years: data from an unselected nationwide study in Denmark. Heart Rhythm 2014;11:1673-1681.2014/05/28.DOI:10.1016/j.hrthm. 2014.05.026.

13. Vetter VL, Dugan N, Guo R, et al. A pilot study of the feasibility of heart screening for sudden cardiac arrest in healthy children. Am Heart J 2011;161:1000-1006e1003.2011/05/17.DOI:10.1016/j.ahj. 2011.01.022.

14. Finocchiaro G, Papadakis M, Robertus J-L, et al. Etiology of Sudden Death in Sports: Insights From a United Kingdom Regional Registry. Journal of the American College of Cardiology 2016;67:108-2115. DOI:https://doi.org/10.1016/j.jacc.2016.02.062. 
15. Bohm P, Scharhag J and Meyer T. Data from a nationwide registry on sports-related sudden cardiac deaths in Germany. Eur J Prev Cardiol 2016; 23: 649-656. 2015/07/02. DOI: 10.1177/2047487315594087.

16. Marijon E, Tafflet M, Celermajer DS, et al. Sports-related sudden death in the general population. Circulation 2011;124:72-681. 2011/07/27.DOI:10.1161/CIRCULATIONAHA.110.008979.

17. Loeys BL, Dietz HC, Braverman AC, et al. The revised Ghent nosology for the Marfan syndrome. 2010;47:476-485.DOI:10.1136/jmg. 2009.072785\%J Journal of Medical Genetics.

18. Baggish AL, Hutter AM, Jr., Wang F, et al. Cardiovascular screening in college athletes with and without electrocardiography: A cross-sectional study. Annals of internal medicine 2010;152:269-275. 2010/03/03.DOI:10.7326/0003-4819-152-5-201003020-00004.

19. Harmon KG, Zigman M and Drezner JA. The effectiveness of screening history, physical exam, and ECG to detect potentially lethal cardiac disorders in athletes: A systematic review/meta-analysis. Journal of Electrocardiology 2015;48:329-338.DOI:https://doi.org/10.1016/j.jelectrocard.2015.02.001.

20. Dhutia H, Malhotra A, Parpia S, et al. The prevalence and significance of a short QT interval in 18825 low-risk individuals including athletes. British Journal of Sports Medicine 2016;50:124-129.DOI:10.1136/bjsports-2015-094827.

21. Priori SG, Blomstrom-Lundqvist C, Mazzanti A, et al. 2015 ESC Guidelines for the management of patients with ventricular arrhythmias and the prevention of sudden cardiac death: The Task Force for the Management of Patients with Ventricular Arrhythmias and the Prevention of Sudden Cardiac Death of the European Society of Cardiology (ESC). Endorsed by: Association for European Paediatric and Congenital Cardiology (AEPC). Eur Heart J 2015;36:2793-2867. 2015/09/01.DOI:10.1093/eurheartj/ehv316.

22. Ryan MP, Cleland JG, French JA, et al. The standard electrocardiogram as a screening test for hypertrophic cardiomyopathy. The American journal of cardiology 1995;76:689-694.1995/10/01.

23. Charron P, Dubourg O, Desnos M, et al. Diagnostic value of electrocardiography and echocardiography for familial hypertrophic cardiomyopathy in genotyped children. Eur Heart J 1998;19:1377-1382. 1998/10/29.

24. Marcus FI, McKenna WJ, Sherrill D, et al. Diagnosis of arrhythmogenic right ventricular cardiomyopathy/dysplasia: proposed modification of the Task Force Criteria. Eur Heart J 2010;31:806-814. 2010/02/23.DOI:10.1093/eurheartj/ehq025.

25. Caselli S, Attenhofer Jost CH, Jenni R, et al. Left Ventricular Noncompaction Diagnosis and Management Relevant to Pre-participation Screening of Athletes. Am J Cardiol 2015;116:801-808.2015/07/05. DOI:10.1016/j.amjcard.2015.05.055.

26. Maron BJ, Friedman RA, Kligfield P, et al. Assessment of the 12-lead ECG as a screening test for detection of cardiovascular disease in healthy general populations of young people (12-25 Years of Age): a scientific statement from the American Heart Association and the American College of Cardiology. Circulation 2014;130:1303-1334. 2014/09/17.DOI:10.1161/CIR.0000000000000025.

27. Sheikh N, Papadakis M, Ghani S, et al. Comparison of electrocardiographic criteria for the detection of cardiac abnormalities in elite black and white athletes. Circulation 2014;129:1637-1649.2014/03/13.DOI: 10.1161/circulationaha.113.006179.

28. Sharma S, Drezner JA, Baggish A, et al. International recommendations for electrocardiographic interpretation in athletes. European heart journal 2018;39:1466-1480.2017/03/23.DOI:10.1093/eurheartj/ehw631.

29. Dhutia H, Malhotra A, Finocchiaro G, et al. Impact of the International Recommendations for Electrocardiographic Interpretation on Cardiovascular Screening in Young Athletes. J Am Coll Cardiol 2017;70: 805-807.2017/08/05.DOI:10.1016/j.jacc.2017.06.018.

30. James CA, Bhonsale A, Tichnell C, et al. Exercise increases age-related penetrance and arrhythmic risk in arrhythmogenic right ventricular dysplasia/cardiomyopathy-associated desmosomal mutation carriers. J Am Coll Cardiol 2013;62:1290-1297.2013/07/23.DOI: 10.1016/j.jacc.2013.06.033. 
31. La Gerche A, Burns AT, Mooney DJ, et al. Exercise-induced right ventricular dysfunction and structural remodelling in endurance athletes. European heart journal 2012;33:998-1006.2011/12/14.DOI: 10.1093/eurheartj/ehr397.

32. Grani C, Benz DC, Schmied C, et al. Prevalence and characteristics of coronary artery anomalies detected by coronary computed tomography angiography in 5634 consecutive patients in a single centre in Switzerland. Swiss Med Wkly 2016;146:w14294.2016/04/29.DOI: 10.4414/smw.2016.14294.

33. Basso C, Maron BJ, Corrado D, et al. Clinical profile of congenital coronary artery anomalies with origin from the wrong aortic sinus leading to sudden death in young competitive athletes. J Am Coll Cardiol 2000;35:1493-1501.2000/05/12.

34. Lorber R, Srivastava S, Wilder TJ, McIntyre S, DeCampli WM, Williams WG, et al. Anomalous Aortic Origin of Coronary Arteries in the Young: Echocardiographic Evaluation With Surgical Correlation. JACC: Cardiovascular Imaging. 2015;8(11):1239-49.

35. Anderson JB, Grenier M, Edwards NM, et al. Usefulness of Combined History, Physical Examination, Electrocardiogram, and Limited Echocardiogram in Screening Adolescent Athletes for Risk for Sudden Cardiac Death. American Journal of Cardiology 2014;114:1763-1767. DOI:10.1016/j.amjcard.2014.09.011.

36. Conroy RM, Pyorala K, Fitzgerald AP, et al. Estimation of ten-year risk of fatal cardiovascular disease in Europe: the SCORE project. Eur Heart J 2003;24:987-1003.2003/06/06.

37. Piepoli MF, Hoes AW, Agewall S, et al. 2016 European Guidelines on cardiovascular disease prevention in clinical practice: The Sixth Joint Task Force of the European Society of Cardiology and Other Societies on Cardiovascular Disease Prevention in Clinical Practice (constituted by representatives of 10 societies and by invited experts)Developed with the special contribution of the European Association for Cardiovascular Prevention and Rehabilitation; Rehabilitation (EACPR). European Heart Journal 2016;37:2315-2381.DOI:10.1093/eurheartj/ehw106\%J European Heart Journal.

38. Thompson PD, Buchner D, Pina IL, et al. Exercise and physical activity in the prevention and treatment of atherosclerotic cardiovascular disease: a statement from the Council on Clinical Cardiology (Subcommittee on Exercise, Rehabilitation, and Prevention) and the Council on Nutrition, Physical Activity, and Metabolism (Subcommittee on Physical Activity). Circulation 2003;107:3109-3116.2003/06/25.DOI: 10.1161/01.CIR.0000075572.40158.77.

39. Gianrossi R, Detrano R, Mulvihill D, et al. Exercise-induced ST depression in the diagnosis of coronary artery disease. A meta-analysis. Circulation 1989;80:87-98.1989/07/01.

40. Albert CM, Mittleman MA, Chae CU, et al. Triggering of sudden death from cardiac causes by vigorous exertion. N Engl J Med 2000; 343:1355-1361.2000/11/09.DOI:10.1056/NEJM200011093431902.

41. Piepoli MF, Hoes AW, Agewall S, et al. 2016 European Guidelines on cardiovascular disease prevention in clinical practice: The Sixth Joint Task Force of the European Society of Cardiology and Other Societies on Cardiovascular Disease Prevention in Clinical Practice (constituted by representatives of 10 societies and by invited experts)Developed with the special contribution of the European Association for Cardiovascular Prevention \&amp; Rehabilitation (EACPR). European Heart Journal 2016;37:2315-2381.DOI:10.1093/eurheartj/ehw106\%J European Heart Journal.

42. Lloyd-Jones DM, Leip EP, Larson MG, et al. Prediction of lifetime risk for cardiovascular disease by risk factor burden at 50 years of age. Circulation 2006;113:791-798.2006/02/08.DOI:10.1161/CIRCULATIONAHA.105.548206.

43. Vlachopoulos C, Xaplanteris P, Aboyans V, et al. The role of vascular biomarkers for primary and secondary prevention. A position paper from the European Society of Cardiology Working Group on peripheral circulation: Endorsed by the Association for Research into Arterial Structure and Physiology (ARTERY) Society. Atherosclerosis 2015;241:507-532.DOI:10.1016/j.atherosclerosis.2015.05.007.

44. Merghani A, Maestrini V, Rosmini S, et al. Prevalence of Subclinical Coronary Artery Disease in 
Masters Endurance Athletes With a Low Atherosclerotic Risk Profile. Circulation 2017; 136: 126-137. 2017/05/04. DOI: 10.1161/CIRCULATIONAHA.116.026964.

45. Dores H, de Araujo Goncalves P, Monge J, et al. Subclinical coronary artery disease in veteran athletes: is a new preparticipation methodology required? Br J Sports Med 2018 2018/11/11.DOI:10.1136/bjsports-2018-099840.

46. Chow BJW, Small G, Yam Y, et al. Prognostic and Therapeutic Implications of Statin and Aspirin Therapy in Individuals With Nonobstructive Coronary Artery Disease. Arteriosclerosis, Thrombosis, and Vascular Biology 2015;35:981-989.DOI:10.1161/ATVBAHA.114. 304351.

47. Laukkanen JA, Rauramaa R, Salonen JT, et al. The predictive value of cardiorespiratory fitness combined with coronary risk evaluation and the risk of cardiovascular and all-cause death. J Intern Med 2007; 262:263-272.2007/07/25.DOI:10.1111/j.1365-2796.2007.01807.x.

48. Dhutia H, Malhotra A, Yeo TJ, et al. Inter-Rater Reliability and Downstream Financial Implications of Electrocardiography Screening in Young Athletes. Circ Cardiovasc Qual Outcomes 2017;10:e003306. 2017/08/16.DOI:10.1161/CIRCOUTCOMES.116.003306. 